\section{Research Square}

Preprints are preliminary reports that have not undergone peer review.

They should not be considered conclusive, used to inform clinical practice, or referenced by the media as validated information.

\title{
Real-world Concordance in Microsatellite Instability Status Between PCR- based Testing and Next-Generation Sequencing Assay for Solid Tumors
}

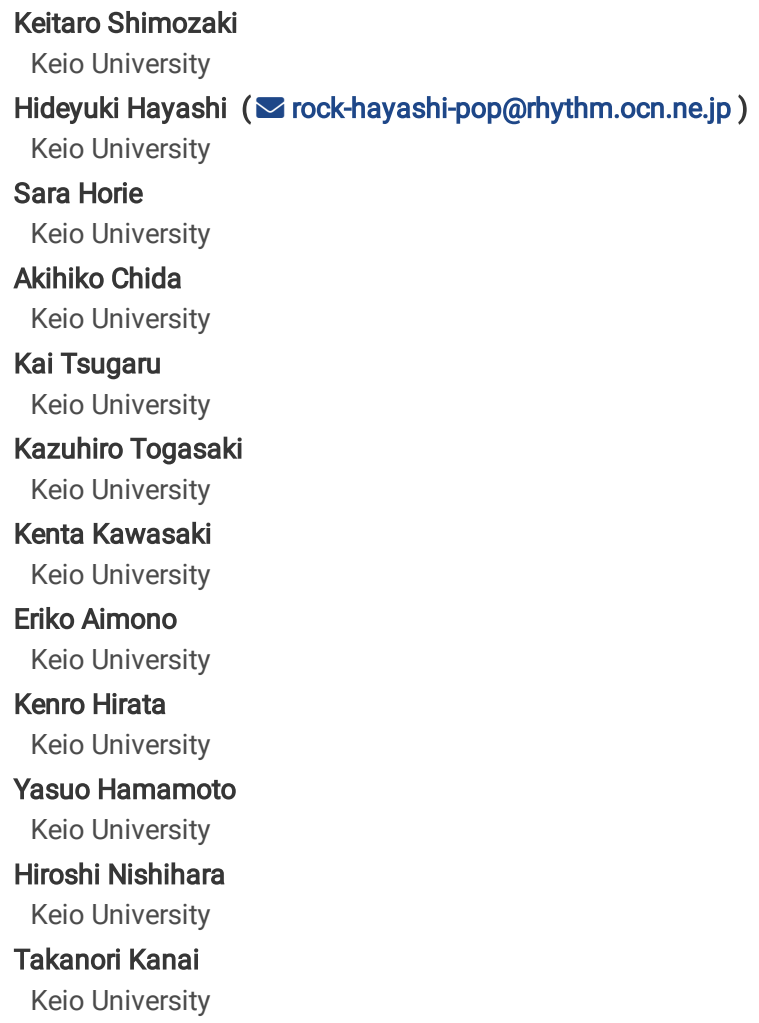

Research Article

Keywords: clinical cancer research, genome-wide sequencing, DNA repair, medical oncology

Posted Date: May 28th, 2021

DOl: https://doi.org/10.21203/rs.3.rs-558267/v1

License: @ (1) This work is licensed under a Creative Commons Attribution 4.0 International License. Read Full License 


\section{Abstract}

Background: Various malignancies exhibit high microsatellite instability (MSI-H) or mismatch repair deficiency (dMMR). The MSI-IVD kit, which can detect MSI status using a polymerase chain reaction (PCR)-based method, was approved as the first tumor-agnostic companion diagnostic for pembrolizumab in patients with MSI-H solid tumors in Japan. Recently, next-generation sequencing (NGS), which can also detect MSI-H/dMMR, has been made clinically available. However, the real-world concordance in MSI-H/dMMR between the PCR-based testing and NGS is yet to be thoroughly investigated.

Methods: A retrospective study was conducted to evaluate the utility of MSI testing using PCR-based testing and NGS assay in patients eligible for both MSI testing and any NGS platform. Co-primary endpoints included positive and negative predictive values of MSI-H/dMMR.

Results: Between December 2018 and June 2020, 40 patients underwent both PCR-based MSI testing and NGS assay for MSI. Two patients with gastric neuroendocrine carcinoma and ovarian cancer were confirmed to have MSI-H/dMMR in both examinations. Among the 38 patients diagnosed as microsatellite stable by PCR-based testing, 2 (5.2\%) with pancreatic cancer were diagnosed as MSI-H after NGS analyses. NGS had positive and negative predictive values of $100 \%(2 / 2)$ and $94.7 \%$ (36/38), respectively, for MSI-H, while the concordance between NGS and PCR-based testing was $94.9 \%$ (38/40).

Conclusion: Similar to PCR-based MSI testing, NGS can be useful for evaluating MSI/MMR status in clinical practice and could be an important alternative method for detecting MSI-H/dMMR in the future.

\section{Introduction}

Recently, immunotherapy has emerged as a new standard of care for various malignancies; however, only a few patients have benefited from antiprogrammed cell death 1 (PD-1)/programmed cell death ligand 1 (PD-L1) and anticytotoxic T lymphocyte antigen 4 (CTLA-4) blockade. High microsatellite instability (MSI$\mathrm{H}$ ) and deficient mismatch repair (dMMR) have been among the established biomarkers for predicting response to immunotherapy, regardless of tumor types (1). Approximately $1-20 \%$ of various advanced malignancies have exhibited MSI-H/dMMR associated with specific clinicopathological, genomic, or prognostic features (2-6). A deficiency in the mismatch repair pathway has been known to induce MSI, an accumulation of DNA replication errors, particularly in genome areas with short repetitive nucleotide sequences. dMMR is indicated by the loss of function of MLH1, MSH2, MSH6, or PMS2 proteins, leading to the loss of function of the mismatch repair pathway, which plays a key role in maintaining genomic stability (7). MSI-H/dMMR tumors produce an increasing number of mutations and neoantigens. $C D 8^{+} \mathrm{T}$ cells recognize these neoantigens, resulting in immune cell infiltration into tumors higher than microsatellite stable (MSS) or proficient MMR (pMMR) tumors (8). Recently, Le et al. had reported that patients with MSI-H/dMMR cancers had robust responses to anti-PD1 antibody $(3,9)$. Given the promising results, the US Food and Drug Administration (FDA) approved pembrolizumab, a humanized IgG4 monoclonal antibody, for the treatment of patients with unresectable or metastatic MSI-H/dMMR solid tumors (10).

The MSI-IVD kit (FALCO Biosystems, Kyoto, Japan), which can detect MSI status using a polymerase chain reaction (PCR)-based method, had been the first approved tumor-agnostic companion diagnostic for pembrolizumab in patients with MSI-H solid tumors in 2018 (11). PCR-based testing determines MSI status using DNA extracted from tumor tissues without using blood samples as reference and analyzes five mononucleotide repeat markers (BAT25, BAT26, MONO27, NR21, and NR24) less susceptible to genetic polymorphisms. The lengths of PCR products from normal DNA are almost confined within the quasimonomorphic variation range (QMVR) (12). The concordance of PCR-based testing and the standard method using both tumor and normal tissue DNA was evaluated and showed complete consistency $(12,13)$. Studies have suggested the existence of possible differences in microsatellite marker length according to tumor type. Considering that the PCR-based testing evaluates each microsatellite marker based on an QMVR width of \pm 3 bases, a slight movement in the wave of each microsatellite marker may result in false-negative results in some solid tumors (14). Moreover, reports have found differences in the concordance between PCR-based testing and immunohistochemistry (IHC) staining according to tumor types, such as brain tumors,

cholangiocarcinoma, ovary cancer, and endometrial cancer (15-17).

Recently, next-generation sequencing (NGS) has emerged as an essential tool for not only detecting genomic alterations of targeted treatment, but also for precise clinical decision-making, including risk assessment, diagnosis, and prognosis. Some NGS platforms, irrespective of the types of commercial or noncommercial base, can also determine MSI status, dMMR, or both. However, differences in the methods used to determine MSI-H have been noted across NGS platforms according to the microsatellite markers adopted and algorithm for deciding MSI-H used. Importantly, discrepancies in MSI status measured using PCR-based testing, NGS, or dMMR measured through IHC staining have been observed. While $\backslash$ tilde $96 \%$ concordance between PCR-based testing and IHC staining has been reported, the correlation between PCR-based testing and NGS is yet to be thoroughly investigated $(16,18)$. Although several studies have reported the concordance between PCR-based testing and NGS and suggested a favorable concordance of $99.4 \%(19,20)$, there are few reports evaluating the real-world concordance of MSI status in PCR-based testing and NGS using data from clinical practice. Given that MSI-H is an established predictive biomarker for immune checkpoint inhibitors, misdiagnosis might influence the therapeutic strategy of the patients who would benefit from immune checkpoint blockade. In Europe and the US, IHC staining has also been recommended for detecting MSI-H/dMMR, which is backed by clinical trials (21). In Japan, however, PCR-based MSI testing has been the only approved companion diagnostic for pembrolizumab. The current study investigated the concordance between PCR-based testing and NGS and determined the utility of NGS in evaluating MSI-H/dMMR using real-world data in Japan.

\section{Patients And Methods}

\section{Patients}

Patients with solid tumors who underwent both PCR-based testing and NGS of any platform and were evaluated for MSI status between July 2015 and May 2020 at Keio University Hospital were retrospectively analyzed. Patients who underwent NGS analysis using NCC Oncopanel (Sysmex Corporation, Kobe, Loading [MathJax]/jax/output/CommonHTML/jax.js $\mathrm{rm}$ could not evaluate MSI status and alterations in MSH6 and PMS2. 


\section{MSI analysis with PCR-based testing}

Tumor DNA from deparaffinized cells was analyzed via PCR using five monomorphic mononucleotide repeat markers (BAT25, BAT26, NR-21, NR-24, and MONO-27) developed by the Promega MSI analysis system (Promega Corporation, Madison, WI). Tumors were classified as MSI-H when two or more of the five markers were positive for shifts in the allelic bands; tumors with one unstable marker were respectively classified as MSI-L and as MSS when none or one of them were positive (12).

\section{MSI analysis with NGS}

FoundationOne CDx (F1CDx) (Foundation Medicine, Cambridge, MA) was approved for all solid tumors in 2019 by the Pharmaceuticals and Medical Devices Agency, a regulatory authority in Japan. Patients with solid tumors who are unresponsive to the standard of care but are eligible for chemotherapy are candidates for this analysis. The testing can be also performed in pediatric patients with cancer or patients with orphan cancers as part of the diagnostic process and for developing treatment strategies on the basis of genomic mutation findings. F1CDx can detect substitutions, insertion and deletion alterations, and copy number alterations across 324 genes, selected gene rearrangements, and tumor mutational burden (TMB) using DNA extracted from formalin-fixed paraffin-embedded (FFPE) tumor tissue specimens (22). To determine MSI status, 95 intronic homopolymer repeat loci (10-20 bp long in the human reference genome) with adequate coverage on the F1CDx Assay were analyzed for length variability and compiled into an overall MSI score via principal components analysis (23). Using the 95 loci, for each sample, the repeat length was calculated in each read that spans the locus and an MSI score was produced. Each sample was assigned a status of MSI-H or MSS; samples with low coverage ( $<250 \times$ median) were assigned a status of MSI-unknown.

PleSSision (Mitsubishi Space Software Co., Ltd., Tokyo, Japan), an outsourcing clinical sequencing system, allows for targeted amplicon exome sequencing of 160 cancer-related genes using the Illumina MiSeq sequencing platform (Illumina, San Diego, CA) (24). This sequencing system evaluated MSI-H/MSS using the MSIsensor program that reports the percentage of unstable microsatellites as a score $(20,25)$. In PleSSision, an MSIsensor score $\geq 20$ is defined as $\mathrm{MSI}-\mathrm{H}$ and $<20$ as MSS.

\section{MMR analysis with immunohistochemistry staining}

The tumor tissues that were categorized as MSI-H by PCR-based testing or NGS were analyzed by immunohistochemistry. Processed IHC slides were evaluated by two pathologists. Cases were defined as dMMR when there was loss of at least one expression of MLH1, MSH2, MSH6, or PMS2 in tumor cells. Considering the immunostaining topographic heterogeneity, the IHC results of MSH2/MSH6 and MLH1/PMS2 patterns were confirmed because MMR proteins function as heterodimers. pMMR was defined as a positive nuclear staining of all MMR proteins.

\section{Statistical analyses}

The positive predictive value for NGS against PCR-based testing was calculated as the proportion of the number of patients who were categorized as MSI-H by NGS divided by the number of patients who were considered as MSI-H by PCR-based testing. The negative predictive value for NGS against PCR-based testing was calculated as the proportion of the number of patients who were categorized as MSS by NGS divided by the number of patients who were considered as MSI-L/MSS by PCR-based testing. Concordance between NGS and PCR-based testing was calculated as the proportion of the number of patients with MSI-H or MSI-L/MSS categorized by both NGS and PCR-based testing divided by the number of all patients. The $95 \%$ confidence interval (Cl) for binomial proportion was calculated based on the exact binomial distribution. The frequency and percentage of tumor samples with concordant and discordant MMR status based on PCR-based testing and NGS results were calculated, after which the extent of concordance was tested using Cohen $\mathrm{k}$ correlation coefficient ( $\mathrm{k}$ ) with its $95 \%$ $\mathrm{Cl}$, the maximum value $\mathrm{k}$ ( $\mathrm{kmax}$ ) given the observed distribution, and exact p-value. All statistical analyses were performed using JMP version 14.2 .0 software (SAS Institute, Cary, NC).

\section{Ethical approval statement}

This study was approved by the Keio University Hospital Institutional Ethics Committee (approval number: 20200046) and was performed in accordance with the Declaration of Helsinki and Ethical Guidelines for Medical and Health Research Involving Human Subjects. Written informed consent was obtained from all patients.

\section{Results}

\section{Patient characteristics}

During July 2015-May 2020, 323 patients underwent PCR-based MSI testing, among whom 63 also underwent NGS. After excluding 23 patients who were evaluated using NCC Oncopanel, 40 patients were ultimately included in the present study. A consort flow diagram is presented in Fig. 1. The median age was 62 (range, 27-89) years, with 17 (43\%) male patients. Moreover, 22 patients (55\%) were evaluated using F1CDx, whereas 18 (45\%) were measured by PleSSision, respectively. The most frequently included tumors were pancreatic ductal carcinoma $(n=9)$, endometrial carcinoma $(n=4)$, extramammary Paget's disease $(n=4)$, ovarian cancer $(n=4)$, cervical cancer $(n=3)$, colorectal cancer $(n=3)$, and sarcoma $(n=3)$. Patient characteristics are described in Table 1 . 
Table 1

Characteristics of the 40 patients recruited for evaluation

\begin{tabular}{|c|c|}
\hline Characteristics & $\begin{array}{l}\text { Patients } \\
(\mathrm{N}=40)\end{array}$ \\
\hline \multicolumn{2}{|l|}{ Age (years) } \\
\hline Median (Range) & $62(27-89)$ \\
\hline \multicolumn{2}{|l|}{ Sex, no (\%) } \\
\hline Male & $17(43 \%)$ \\
\hline Female & $23(57 \%)$ \\
\hline \multicolumn{2}{|l|}{ Types of NGS, no (\%) } \\
\hline FoundationOne CDx & $22(55 \%)$ \\
\hline PleSSision & $18(45 \%)$ \\
\hline \multicolumn{2}{|l|}{ Primary sites, no (\%) } \\
\hline Pancreas & $9(23 \%)$ \\
\hline Endometrial & $4(10 \%)$ \\
\hline Extramammary Paget's disease & $4(10 \%)$ \\
\hline Ovary & $4(10 \%)$ \\
\hline Cervix & $3(8 \%)$ \\
\hline Colorectal & $3(8 \%)$ \\
\hline Sarcoma & $3(8 \%)$ \\
\hline Biliary & $2(5 \%)$ \\
\hline Esophageal & $1(3 \%)$ \\
\hline Head and neck & $1(3 \%)$ \\
\hline Neuroendocrine carcinoma & $1(3 \%)$ \\
\hline Olfactory neuroblastoma & $1(3 \%)$ \\
\hline Peritoneal & $1(3 \%)$ \\
\hline Stomach & $1(3 \%)$ \\
\hline Thyroid & $1(3 \%)$ \\
\hline Unknown primary & $1(3 \%)$ \\
\hline
\end{tabular}

Abbreviations: NGS, next-generation sequencing.

\section{MSI status according to PCR-based testing and NGS}

Among 40 included patients, 2 (5\%) with gastric neuroendocrine carcinoma (50-year-old male patient) and ovarian cancer (60-year-old female patient) were determined as MSI-H by both PCR-based testing and NGS assay. NGS detected MSH2 mutation and PMS2 mutation in the former and latter, respectively. IHC staining results were consistent with those of NGS, i.e., loss of MSH2 and MSH6 was observed in the former, whereas loss of PMS2 was observed in the latter.

Among 38 patients who were initially categorized as MSI-L/MSS by PCR-based testing, 2 (5.3\%) with pancreatic ductal carcinoma were confirmed as MSI-H and dMMR by NGS. NGS identified MSH2 and MLH1 mutation in these patients, respectively. These two patients (a 47-year-old female with loss of MSH2 and MSH6 and a 78-year-old female with loss of MLH1 and PMS2) were also evaluated by IHC staining, which confirmed dMMR (Fig. 2). Both had already been diagnosed with Lynch syndrome and had family histories of cancer.

An expert panel discussed each NGS assay result. Genotype-matched treatment was provided to three patients, all of whom received pembrolizumab. Moreover, two partial responses were observed. The characteristics of all four cases determined to be MSI-H in this study are described in Table 2. 
Table 2

Details for the four cases with deficient mismatch repair and/or high microsatellite instability confirmed by polymerase chain reaction-based testing and/or next-generation sequencing

\begin{tabular}{|c|c|c|c|c|c|c|c|c|c|c|c|c|}
\hline \multirow[t]{2}{*}{ No } & \multirow[t]{2}{*}{ Age } & \multirow[t]{2}{*}{ Sex } & \multirow{2}{*}{$\begin{array}{l}\text { Primary } \\
\text { site }\end{array}$} & \multirow{2}{*}{$\begin{array}{l}\text { Past } \\
\text { Medical } \\
\text { history }\end{array}$} & \multirow{2}{*}{$\begin{array}{l}\text { Family } \\
\text { histories }\end{array}$} & \multirow{2}{*}{$\begin{array}{l}\text { PCR } \\
\text { Result }\end{array}$} & \multicolumn{2}{|l|}{ NGS } & $\mathrm{IHC}$ & \multirow{2}{*}{$\begin{array}{l}\text { TMB } \\
\text { (mut/Mb) }\end{array}$} & \multirow{2}{*}{$\begin{array}{l}\text { Actionable } \\
\text { gene } \\
\text { alterations* }\end{array}$} & \multirow{2}{*}{$\begin{array}{l}\text { Use of immune } \\
\text { checkpoint } \\
\text { blockade, } \\
\text { Best response }\end{array}$} \\
\hline & & & & & & & Platform & Result & Result & & & \\
\hline 1 & 50 & $M$ & gNEC & None & $\begin{array}{l}\text { Primary } \\
\text { unknown: } \\
\text { aunt }\end{array}$ & MSI-H & PleSSision & MSI-H & $\begin{array}{l}\text { MSH2 } \\
\text { and } \\
\text { MSH6 } \\
\text { loss }\end{array}$ & 52.4 & $\begin{array}{l}M S H 2 \\
\text { p.Y408Ffs*5, } \\
\text { GNAS } \\
\text { p.R844H }\end{array}$ & $\begin{array}{l}\text { Pembrolizumab } \\
\text { PR }\end{array}$ \\
\hline 2 & 60 & $\mathrm{~F}$ & Ovary & None & $\begin{array}{l}\text { Colorectal: } \\
\text { mother, } \\
\text { Lung: } \\
\text { grandfather, } \\
\text { Liver: } \\
\text { grandfather }\end{array}$ & MSI-H & PleSSision & MSI-H & $\begin{array}{l}\text { PMS2 } \\
\text { loss }\end{array}$ & 18.8 & $\begin{array}{l}\text { PMS2 } \\
\text { p.R315*, } \\
\text { PIK3CA } \\
\text { p.C378R, NF1 } \\
\text { p.Y628Lfs*6, } \\
\text { ARIDA1A } \\
\text { p.S617Qfs*2 }\end{array}$ & $\begin{array}{l}\text { Pembrolizumab } \\
\text { NE }\end{array}$ \\
\hline 3 & 47 & $\mathrm{~F}$ & Pancreas & $\begin{array}{l}\text { Lynch } \\
\text { syndrome: } \\
\text { Colorectal; } \\
\text { Endometrial; } \\
\text { Ovary }\end{array}$ & $\begin{array}{l}\text { Lung: father, } \\
\text { uncle; } \\
\text { Stomach: } \\
\text { uncle; } \\
\text { Adrenal } \\
\text { gland: } \\
\text { grandmother }\end{array}$ & MSS & PlesSision & MSI-H & $\begin{array}{l}\text { MSH2 } \\
\text { and } \\
\text { MSH6 } \\
\text { loss }\end{array}$ & 13.4 & $\begin{array}{l}\text { MSH2 } \\
\text { p.T788Nfs*11, } \\
\text { KRASp.Q61R, } \\
\text { GNASR } \\
\text { p.844C }\end{array}$ & $\begin{array}{l}\text { Pembrolizumab } \\
\text { PR }\end{array}$ \\
\hline 4 & 78 & $\mathrm{~F}$ & Pancreas & $\begin{array}{l}\text { Lynch } \\
\text { syndrome }\end{array}$ & $\begin{array}{l}\text { Pancreas: } \\
\text { mother; } \\
\text { Stomach: } \\
\text { sister, } \\
\text { grandmother }\end{array}$ & MSS & PleSSision & MSI-H & $\begin{array}{l}M L H 1 \\
\text { and } \\
P M S 2 \\
\text { loss }\end{array}$ & 45.6 & $\begin{array}{l}\text { MLH1 } \\
\text { p.R385C, } \\
\text { KRASp.G12D }\end{array}$ & $\begin{array}{l}- \\
\mathrm{NE}\end{array}$ \\
\hline
\end{tabular}

Abbreviations: dMMR, deficient mismatch repair; F, female; gNEC, gastric neuroendocrine carcinoma; IHC, immunohistochemistry; M, male; MSI-H, microsatellite instability high; MSS, microsatellite stable; NE, not evaluable; NGS, next-generation sequencing; PR, partial response; TMB, tumor mutational burden

* Gene alterations denote gene mutations and copy number alteration

Concordance between PCR-based testing and NGS

For MSI-H detection, the positive and negative predictive values of NGS against PCR-based testing were 2/2 [100\%; 95\% Cl, 37.6-100] and 36/38 (94.7\%; $95 \%$ $\mathrm{Cl}, 91.2-94.7)$, respectively. Meanwhile, the concordance rate between PCR-based testing and NGS was 38/40 (94.9\%; 95\% Cl, 88.5-94.9) (Table 3). The MSI-H status assessed by NGS or PCR-based testing and dMMR evaluated by IHC were consistent (100\%), whereas 36 patients categorized as MSS by both NGS and PCR-based testing were not evaluated by IHC in this study.

Table 3

Concordance and discordance in microsatellite instability status between next-generation sequencing and polymerase chain reaction-based testing

\begin{tabular}{|lllll|}
\hline NGS assay & \multicolumn{2}{l}{ PCR-based testing } & \multicolumn{2}{l|}{ Immunohistochemistry staining } \\
\cline { 2 - 5 } & MSI-H $(\mathbf{n = 2})$ & MSI-L/MSS $(\mathbf{n = 3 8 )}$ & dMMR, $\mathbf{n}$ & pMMR, $\mathbf{n}$ \\
\hline MSI-H $(n=4)$ & 2 & 2 & 4 & 0 \\
\hline MSS $(n=36)$ & 0 & 36 & 0 & Not evaluated \\
\hline Positive predictive value of NGS against PCR-based testing & $\mathbf{1 0 0 \% ( 2 / 2 )}$ & \\
\hline Negative predictive value of NGS against PCR-based testing & $\mathbf{9 4 . 7 \% ( 3 6 / 3 8 )}$ & \\
\hline Concordance between NGS and the PCR-based testing & $\mathbf{9 4 . 9 \% ( 3 8 / 4 0 )}$ & \\
\hline
\end{tabular}

Abbreviations: dMMR, deficient mismatch repair; MSI-H, microsatellite instability high; MSI-L, microsatellite instability low; MSS, microsatellite stable; NGS, next-generation sequencing; PCR, polymerase chain reaction; pMMR, proficient mismatch repair

Tissue samples used for PCR-based testing and NGS were consistent in all patients. Twenty-seven patients (68\%) were evaluated using tissues obtained from the primary tumor site, whereas 13 patients (32\%) were examined using biopsied or resected metastatic tissues.

\section{Discussion}


This retrospective study suggested that the sensitivity and specificity of NGS could make it an alternative to PCR-based MSI testing, the approved companion diagnostic for detecting MSI-H in Japan. To the best of our knowledge, this is the first study to evaluate the real-world concordance and discordance between PCR-based testing kit and NGS using clinical data in Japan. Four patients included herein had been identified as MSI-H by PCR-based testing or NGS. Among them, two patients were initially determined as MSS by PCR-based testing and subsequently re-evaluated to be MSI-H by NGS. IHC staining confirmed the deficiency in all MMRs among all four patients. Notably, false negatives in PCR-based MSI testing were suspected in such cases. Generally, reports have shown that low tumor burden or increase in tumor DNA degradation over time observed in the samples could be attributed to false-negative results. Moreover, several studies have reported that Lynch syndrome with germline mutations in MSH6 or PMS2 might not necessarily show MSI-H (26, 27). However, the current study used the same samples during PCR-based testing and NGS assay, with all four patients showing MSI-H in NGS, unlike PCR-based testing.

Our study highlights the accuracy of PCR-based MSI testing as well as the possible usefulness of the NGS assay in clinical practice. Vanderwalde et al. assessed the concordance between PCR-based testing and NGS in 2,189 matched cases of 26 cancer types, including 23 cases with MSI-H. In MSI-H detection, NGS had a sensitivity of $95.8 \%$ (95\% Cl, 92.24-98.08), specificity of $99.4 \%$ (95\% Cl, 98.94-99.69), positive predictive value of $94.5 \%$ (95\% Cl, $90.62-$ 97.14), and negative predictive value of $99.2 \%$ (95\% Cl, 98.75-99.57) in comparison with PCR-based testing (19). Moreover, Middha et al. validated NGS against PCR-based testing and MMR immunohistochemistry for 138 colorectal cancers (CRCs), including 24 MSI-H CRCs, and 40 uterine endometrioid cancers (UECs), including 15 MSI-H UECs, and showed a concordance of 99.4\% (20). Although these studies have reported concordance between MSI status assessed by NGS and PCR-based testing with a larger number of patients in a laboratory setting, our present study demonstrated the clinical utility of NGS to analyze MSI status using real-world data in Japan where PCR-based MSI testing is the only approved companion diagnostic for the use of immune checkpoint blockade as of now. Further, our study was performed in a situation close to real clinical practice where tissue samples are not abundant or inadequate for MSI testing analysis compared with previous reports in which tissue samples were obtained from another study.

NGS can investigate a large variety of gene alterations at one time. Generally, PCR-based MSI testing requires tumor specimens with FFPE block or 5-10 pieces of undyed 5- $\mu \mathrm{m}$ pathological specimens, which is equivalent to specimens required for NGS assay to perform genomic testing. The number of tumor samples had been either limited, particularly in patients with pancreatic carcinoma, prostate cancer, or cancer of unknown primary whose tumor burden is generally low or tumor is difficult to access, such as those obtained through biopsy. Hence, we often experienced problems related to the inability to order new genomic testing kits given the lack of available specimens. However, rebiopsy might be difficult for patients with poor conditions or without superficial metastasis. To address such problems, using NGS initially might be an ideal alternative.

One strength of the NGS assay is its ability to simultaneously detect gene alterations in $M L H 1, M S H 2, M S H 6$, and PMS2.(22, 24) Furthermore, certain types of NGS can also analyze germline mutations associated with Lynch syndrome by assessing matched normal-tumor pairs (28). Although the current NGS assay could not analyze loss of function due to DNA methylation, such an approach could be realized in the near future (29).

By counting thousands of fractional mutations, NGS was able to evaluate TMB. TMB is known to be one of the novel predictive biomarkers for the efficacy of immunotherapy $(30,31)$. Despite the current lack of approval for the use of pembrolizumab among these populations in Japan, the FDA has approved pembrolizumab for patients with unresectable or metastatic TMB-H ( $\geq 10$ mutations/megabase solid tumors by F1CDx in 2020. Although the cutoff of TMB-H still remains controversial, clinicians would greatly benefit from knowing TMB values when considering immunotherapy.

Conversely, NGS does have some problems that need to be considered. First, NGS costs approximately 560,000 yen (5,300 US dollars), whereas PCR-based MSI testing costs 20,000 yen (190 US dollars) under the health insurance system in Japan, placing considerable economic burden on the national insurance system. Second, the average turnaround time (TAT) for NGS is approximately $4-5$ weeks following sample receipt in the laboratory, which might affect treatment decision-making. Furthermore, an expert panel discussion is needed to confirm whether gene alterations are meaningful after the results have returned.

The utility of NGS, including the identification of MSI-H/dMMR status, could supersede that of PCR-based MSI testing provided that cost reductions and shortening of TAT are addressed. Furthermore, the ability of NGS to be performed earlier and at a more adequate timing has made it an indispensable diagnostic strategy. Moreover, forthcoming advancement would allow MSI-H/dMMR to be evaluated using cell-free DNA from a patient's blood sample. Accordingly, FoundationOne Liquid CDx (Foundation Medicine, Cambridge, MA) and Guardant360 CDx (Guardant Health, Redwood City, CA), which are types of novel platforms for liquid biopsy, can detect MSI status and have received approval from the FDA in 2020 (32). Though liquid biopsy, MSI/MMR status can be determined more quickly and effortlessly, allowing us to not only administer immunotherapy for patients with MSI-H/dMMR tumors, but also recruit patients with certain gene alterations into clinical trials (33). We believe that NGS should be performed diligently in patients suspected to be MSI-H/dMMR after considering their clinical course, past medical history, and family history, regardless of whether they have been determined as MSS by PCR-based testing.

Some limitations of the current study inherent to its retrospective nature are worth noting. First, this was a single-center study; the sample size was limited with potential bias in patient selection. However, we believe that determining the concordance between NGS and PCR-based MSI testing using data from clinical practice is crucial. Second, we did not perform IHC staining in all cases determined as MSS by PCR-based testing, potentially underestimating the number of patients with dMMR. However, the percentage of MSI-H/dMMR cases in our study was $10 \%$, comparable to those reported in previous reports. Further, the facilities where NGS can be performed are limited, and the conditions for insurance coverage are strictly limited in Japan. Patients with good performance status who have completed the standard treatment or who are with rare cancers for which a standard treatment has not been yet established are candidates for NGS. Thus, patients who were included in this study might have been biased, particularly with respect to tumor types.

In conclusion, the NGS assay has comparable utility to PCR-based MSI testing for evaluating MSI/MMR status. Thus, NGS can be expected to become an important alternative method for detecting MSI-H/dMMR in the near future after reducing costs, shortening TAT, and improving accessibility at appropriate testing periods.

Loading [MathJax]/jax/output/CommonHTML/jax.js 


\section{Declarations}

\section{Acknowledgments}

The authors thank the patients and their families and caregivers for participating in this study. This work was supported in part by the JSPS (Japan Society for the Promotion of Science) KAKENHI (Grant-in-Aid for Young Scientists B) Grant Number 17K15910.

\section{Funding}

We have no funding to declare.

\section{Author's contributions}

$\mathrm{KS}$ and $\mathrm{HH}$ designed the study. $\mathrm{SH}, \mathrm{AC}, \mathrm{KTs}, \mathrm{KTo}, \mathrm{KK}, \mathrm{EA}, \mathrm{KH}$, and $\mathrm{YH}$ gathered the data. $\mathrm{KS}, \mathrm{HH}$, and $\mathrm{HN}$ analyzed and interpreted the data. $\mathrm{KS}$ wrote the manuscript. All authors were involved in development, review, and approval of the manuscript. KS, HH, and TK were responsible for the final decision for publication.

\section{Ethical approval and consent to participate}

This study was approved by the Keio University Hospital Institutional Ethics Committee (approval number: 20200046) and was performed in accordance with the Declaration of Helsinki and Ethical Guidelines for Medical and Health Research Involving Human Subjects. Written informed consent was obtained from all patients.

\section{Patient consent for publication}

Not applicable.

\section{Conflicts of interest disclosure statement}

The authors have no conflict of interest.

\section{References}

1. Dudley JC, Lin MT, Le DT and Eshleman JR: Microsatellite Instability as a Biomarker for PD-1 Blockade. Clin Cancer Res 22: 813-820, 2016.

2. Popat S, Hubner R and Houlston RS: Systematic review of microsatellite instability and colorectal cancer prognosis. J Clin Oncol 23: 609-618, 2005.

3. Le DT, Durham JN, Smith KN, et al.: Mismatch repair deficiency predicts response of solid tumors to PD-1 blockade. Science 357: 409-413, 2017.

4. Ward R, Meagher A, Tomlinson I, et al.: Microsatellite instability and the clinicopathological features of sporadic colorectal cancer. Gut 48: 821-829, 2001.

5. Cancer Genome Atlas N: Comprehensive molecular characterization of human colon and rectal cancer. Nature 487: 330-337, 2012.

6. Bonneville R, Krook MA, Kautto EA, et al.: Landscape of Microsatellite Instability Across 39 Cancer Types. JCO Precision Oncology: 1-15, 2017.

7. Hause RJ, Pritchard CC, Shendure J and Salipante SJ: Classification and characterization of microsatellite instability across 18 cancer types. Nat Med 22 : 1342-1350, 2016

8. Narayanan S, Kawaguchi T, Peng X, et al.: Tumor Infiltrating Lymphocytes and Macrophages Improve Survival in Microsatellite Unstable Colorectal Cancer. Sci Rep 9: 13455, 2019.

9. Le DT, Uram JN, Wang H, et al.: PD-1 Blockade in Tumors with Mismatch-Repair Deficiency. N Engl J Med 372: 2509-2520, 2015.

10. Marcus L, Lemery SJ, Keegan P and Pazdur R: FDA Approval Summary: Pembrolizumab for the Treatment of Microsatellite Instability-High Solid Tumors. Clin Cancer Res 25: 3753-3758, 2019.

11. Mishima S, Taniguchi H, Akagi K, et al.: Japan Society of Clinical Oncology provisional clinical opinion for the diagnosis and use of immunotherapy in patients with deficient DNA mismatch repair tumors, cooperated by Japanese Society of Medical Oncology, First Edition. Int J Clin Oncol 25: 217-239, 2020.

12. Bando H, Okamoto W, Fukui T, Yamanaka T, Akagi $\mathrm{K}$ and Yoshino $\mathrm{T}$ : Utility of the quasi-monomorphic variation range in unresectable metastatic colorectal cancer patients. Cancer Sci 109: 3411-3415, 2018.

13. Umar A, Boland CR, Terdiman JP, et al.: Revised Bethesda Guidelines for hereditary nonpolyposis colorectal cancer (Lynch syndrome) and microsatellite instability. J Natl Cancer Inst 96: 261-268, 2004.

14. Patil DT, Bronner MP, Portier BP, Fraser CR, Plesec TP and Liu X: A five-marker panel in a multiplex PCR accurately detects microsatellite instability-high colorectal tumors without control DNA. Diagn Mol Pathol 21: 127-133, 2012.

15. Wang Y, Shi C, Eisenberg R and Vnencak-Jones CL: Differences in Microsatellite Instability Profiles between Endometrioid and Colorectal Cancers: A Potential Cause for False-Negative Results? J Mol Diagn 19: 57-64, 2017.

16. Bartley AN, Luthra R, Saraiya DS, Urbauer DL and Broaddus RR: Identification of cancer patients with Lynch syndrome: clinically significant discordances and problems in tissue-based mismatch repair testing. Cancer Prev Res (Phila) 5: 320-327, 2012.

17. Winkelmann R, Schneider M, Hartmann S, et al.: Microsatellite Instability Occurs Rarely in Patients with Cholangiocarcinoma: A Retrospective Study from a German Tertiary Care Hospital. Int J Mol Sci 192018.

Loading [MathJax]/jax/output/CommonHTML/jax.js 
18. Cicek MS, Lindor NM, Gallinger S, et al.: Quality assessment and correlation of microsatellite instability and immunohistochemical markers among population- and clinic-based colorectal tumors results from the Colon Cancer Family Registry. J Mol Diagn 13: 271-281, 2011.

19. Vanderwalde A, Spetzler D, Xiao N, Gatalica Z and Marshall J: Microsatellite instability status determined by next-generation sequencing and compared with PD-L1 and tumor mutational burden in 11,348 patients. Cancer Med 7: 746-756, 2018.

20. Middha S, Zhang L, Nafa K, et al.: Reliable Pan-Cancer Microsatellite Instability Assessment by Using Targeted Next-Generation Sequencing Data. JCO Precis Oncol 20172017.

21. Luchini C, Bibeau F, Ligtenberg MJL, et al.: ESMO recommendations on microsatellite instability testing for immunotherapy in cancer, and its relationship with PD-1/PD-L1 expression and tumour mutational burden: a systematic review-based approach. Ann Oncol 30: 1232-1243, 2019.

22. Schwaederle M, Daniels GA, Piccioni DE, et al.: On the Road to Precision Cancer Medicine: Analysis of Genomic Biomarker Actionability in 439 Patients. Mol Cancer Ther 14: 1488-1494, 2015.

23. https://www.accessdata.fda.gov/cdrh_docs/pdf17/P170019B.pdf.

24. Hayashi H, Tanishima S, Fujii K, et al.: Clinical impact of a cancer genomic profiling test using an in-house comprehensive targeted sequencing system. Cancer Sci2020.

25. Niu B, Ye K, Zhang Q, et al.: MSIsensor: microsatellite instability detection using paired tumor-normal sequence data. Bioinformatics 30: 1015-1016, 2014.

26. Shia J, Zhang L, Shike M, et al.: Secondary mutation in a coding mononucleotide tract in MSH6 causes loss of immunoexpression of MSH6 in colorectal carcinomas with MLH1/PMS2 deficiency. Mod Pathol 26: 131-138, 2013.

27. Clendenning M, Senter L, Hampel H, et al.: A frame-shift mutation of PMS2 is a widespread cause of Lynch syndrome. J Med Genet 45: $340-345$, 2008.

28. Sunami K, Ichikawa H, Kubo T, et al.: Feasibility and utility of a panel testing for 114 cancer-associated genes in a clinical setting: A hospital-based study. Cancer Sci 110: 1480-1490, 2019.

29. Barros-Silva D, Marques CJ, Henrique R and Jeronimo C: Profiling DNA Methylation Based on Next-Generation Sequencing Approaches: New Insights and Clinical Applications. Genes (Basel) 92018.

30. Marabelle A, Le DT, Ascierto PA, et al.: Efficacy of Pembrolizumab in Patients With Noncolorectal High Microsatellite Instability/Mismatch Repair-Deficient Cancer: Results From the Phase II KEYNOTE-158 Study. J Clin Oncol 38: 1-10, 2020.

31. Marabelle A, Fakih M, Lopez J, et al.: Association of tumour mutational burden with outcomes in patients with advanced solid tumours treated with pembrolizumab: prospective biomarker analysis of the multicohort, open-label, phase 2 KEYNOTE-158 study. Lancet Oncol 21: 1353-1365, 2020.

32. The Food and Drug Administration: Premarket Approval (PMA). 2020.

33. Nakamura Y, Taniguchi H, Ikeda M, et al.: Clinical utility of circulating tumor DNA sequencing in advanced gastrointestinal cancer: SCRUM-Japan GISCREEN and GOZILA studies. Nat Med2020.

\section{Figures}




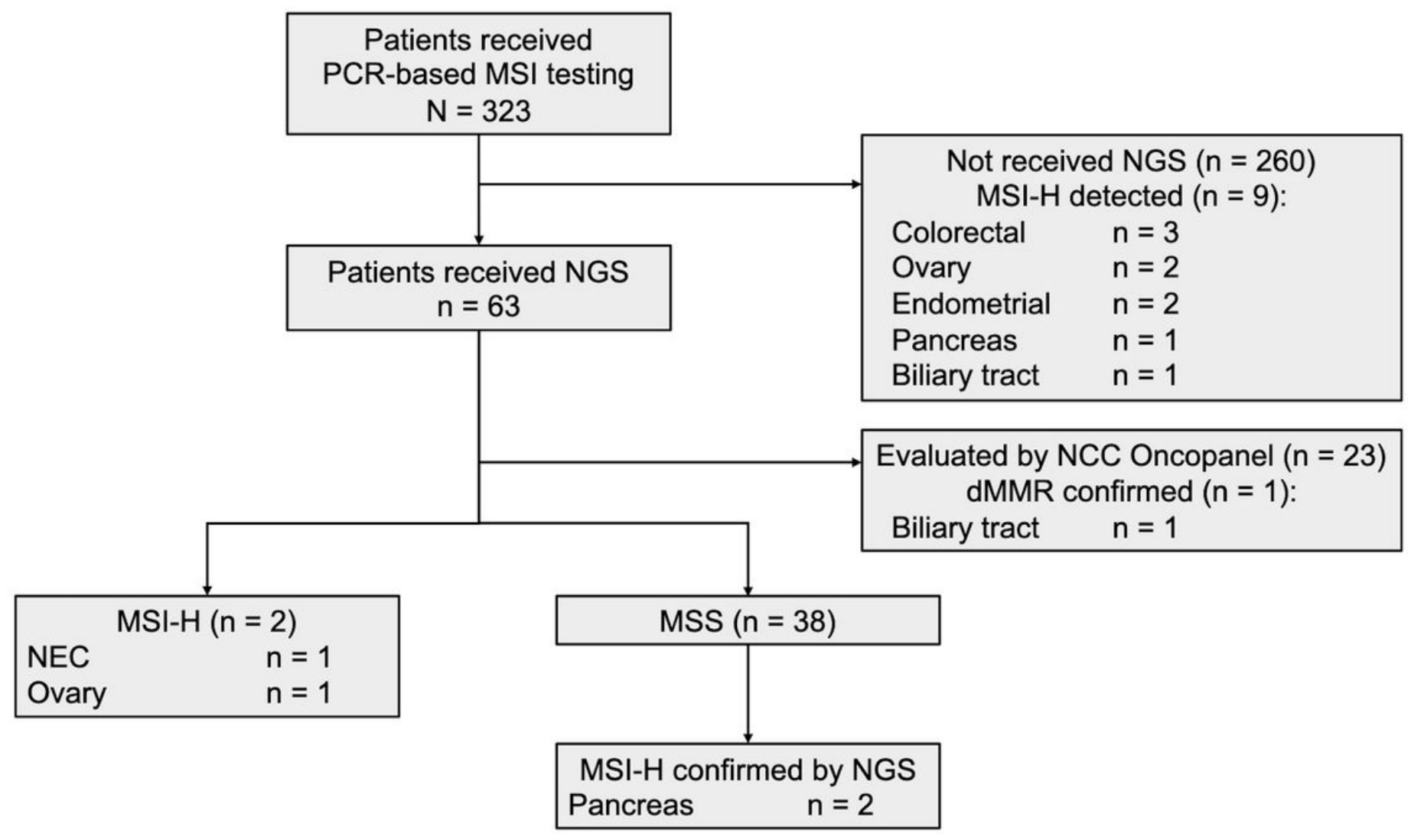

Figure 1

CONSORT diagram of this study Abbreviations: dMMR, deficient mismatch repair; MSI-H, microsatellite instability high; MSS, microsatellite stable; NEC, neuroendocrine carcinoma; NGS, next-generation sequencing

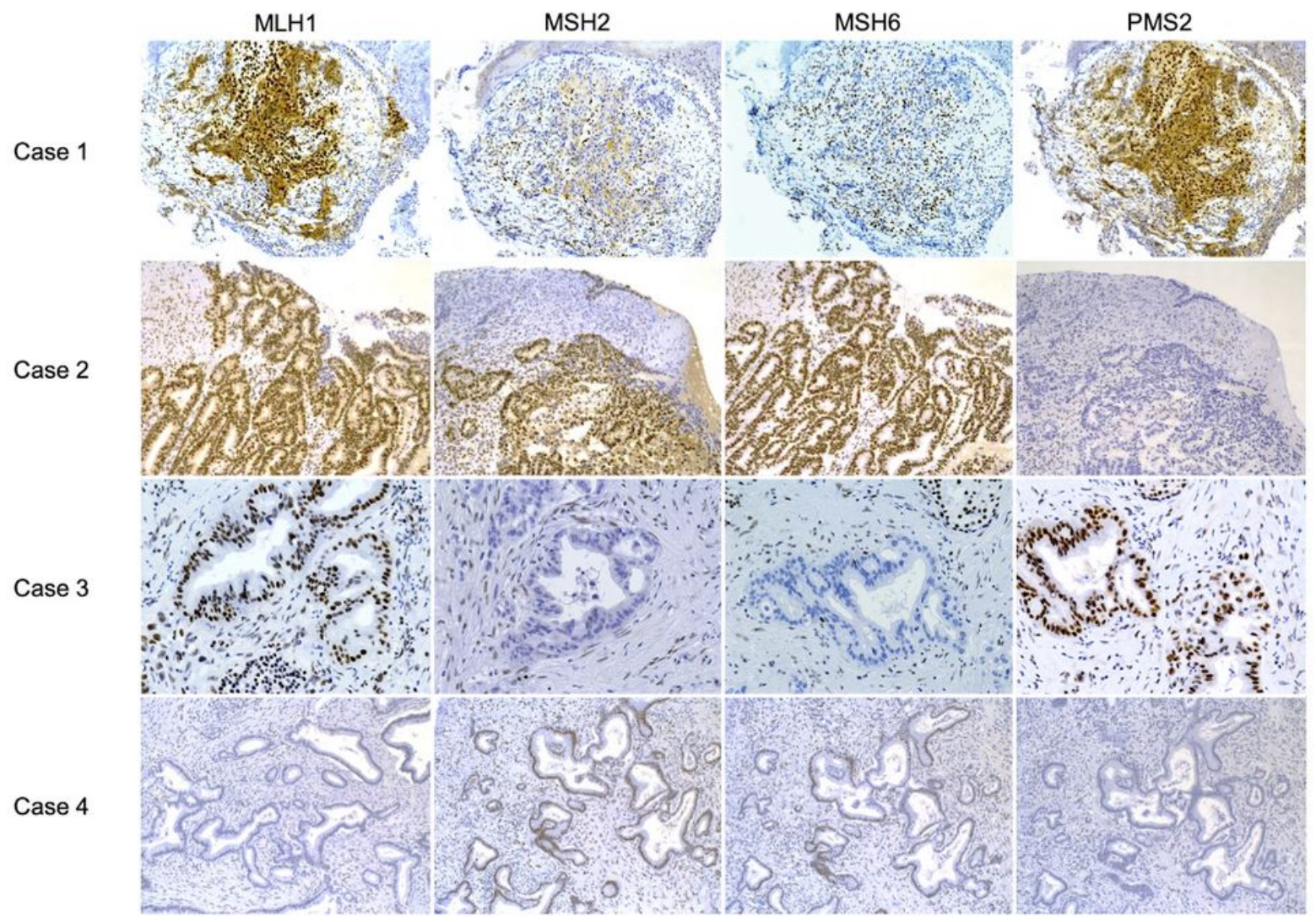


Figure 2

Immunohistochemistry staining of four patients with MSI-H/dMMR solid tumors 